\title{
Satisfação do paciente em um programa educativo para automonitorização da glicemia capilar no domicilio
}

\author{
Patient satisfaction in a home-based capillary blood glucose self-monitoring educational program
}

Satisfacción del paciente en programa educativo de automonitoreo de glucemia capilar en domicilio

Denise Ferro ${ }^{1}$, Vivian Saraiva Veras ${ }^{2}$, Flávia Fernanda Luchetti Rodrigues ${ }^{3}$, Tatiane Aparecida Martins ${ }^{4}$, Carla Regina de Souza Teixeira ${ }^{5}$, Manoel Antônio dos Santos ${ }^{6}$, Maria Lucia Zanetti ${ }^{7}$

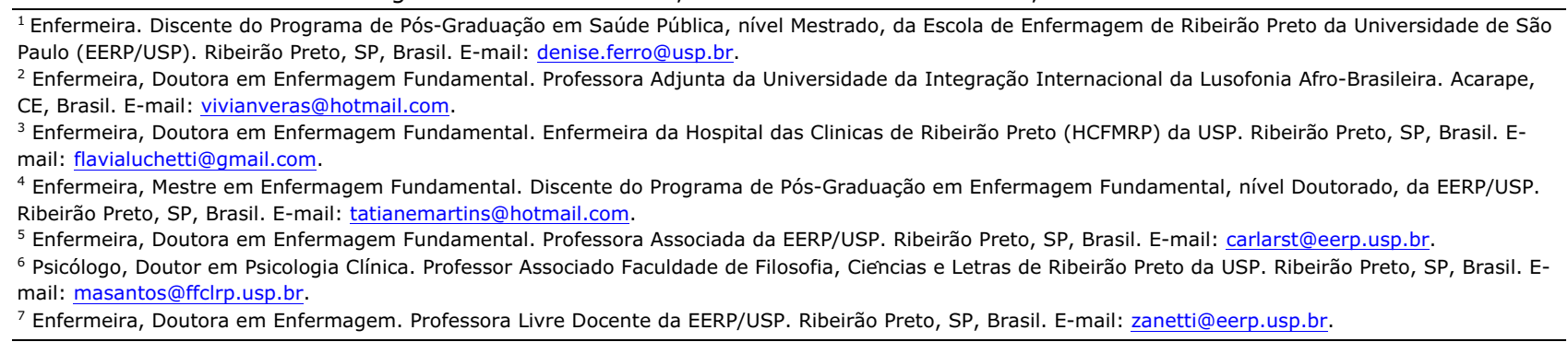

\section{RESUMO}

Estudo transversal que teve como objetivo avaliar a satisfação dos pacientes com diabetes mellitus após oferecimento de um programa educativo para automonitorização da glicemia capilar no domicílio. A amostra por conveniência foi constituída por 29 pacientes de duas Unidades Básicas de Saúde de uma cidade do interior paulista, em 2012. Utilizou-se um formulário contendo as variáveis sociodemográficas e um questionário para avaliar a satisfação dos pacientes em relação ao grupo de educação em diabetes. Os resultados mostraram que a maioria dos pacientes considerou o programa educativo como excelente/satisfatório. Quanto às informações recebidas, os pacientes referiram que elas atenderam às suas necessidades e que recomendariam o programa educativo a outra pessoa. Conclui-se que há necessidade de investimentos para que os programas educativos disponibilizados nos serviços de saúde às pessoas com DM sejam permanentemente avaliados e que utilizem a satisfação como um indicador de avaliação.

Descritores: Diabetes Mellitus; Satisfação do Paciente; Enfermagem.

\section{ABSTRACT}

The aim of this cross-sectional study was to assess the satisfaction of patients with diabetes mellitus (DM) after being provided with an educative program for self-monitoring capillary glucose at home. The convenience sample was made of 29 patients, from two basic health units in a city in the interior of São Paulo, in 2012. Patient satisfaction regarding the diabetes education group was assessed using a questionnaire and a form containing sociodemographic variables. Results showed that most of the patients considered the educative program to be excellent/satisfactory. Regarding the information received, the patients stated it met their needs and that they would recommend the program to other people. In conclusion, investments are needed so that the educative programs provided in health services to people with DM are permanently assessed and use satisfaction as an assessment indicator.

Descriptors: Diabetes Mellitus; Patient Satisfaction; Nursing.

\section{RESUMEN}

Estudio transversal objetivando evaluar la satisfacción de pacientes con diabetes mellitus luego de ofrecérseles un programa educativo de automonitoreo de glucemia capilar en domicilio. Muestra por conveniencia constituida por 29 pacientes de dos Unidades Básicas de Salud de ciudad del interior paulista. Se utilizó un cuestionario incluyendo variable sociodemográficas y otro evaluando la satisfacción de los pacientes respecto del grupo educativo constituido. Los resultados mostraron que la mayoría de los pacientes consideró al programa educativo como excelente/satisfactorio. Respecto a las informaciones recibidas, los pacientes refirieron que las mismas atendieron sus necesidades y que recomendarían el programa educativo a otra persona. Se concluye en que existe necesidad de inversiones para que los programas educativos disponibles en los servicios de salud para personas con DM sean permanentemente evaluados y que utilicen la satisfacción como un factor indicador de la evaluación.

Descriptores: Diabetes Mellitus; Satisfação do Paciente; Enfermería. 


\section{INTRODUÇÃo}

A satisfação do usuário de saúde é percebida como uma meta almejada pelos gestores e equipe de saúde para o aprimoramento do atendimento oferecido pelos serviços $^{(1)}$. O conceito de satisfação privilegia o usuário na avaliação da qualidade em saúde. Há vários modelos de medida, mas todos têm como pressuposto comum abordar as percepções do paciente em relação às suas expectativas, valores e desejos ${ }^{(2)}$.

Satisfação do usuário pode ser definida como a avaliação que cada indivíduo faz das diferentes dimensões do cuidado à saúde ${ }^{(2)}$. Esse construto tem sido objeto de investigação devido às repercussões que pode trazer no incremento da adesão ao tratamento prescrito, além de fornecer informações valiosas para os profissionais de saúde e maior comparecimento aos serviços ${ }^{(1-2)}$.

Estudos internacionais mostram a satisfação dos usuários com diabetes mellitus (DM) em programas de automonitorização ${ }^{(3-4)}$. Pacientes bem controlados mostraram maior satisfação com a frequência de automonitorização no intervalo de seis meses em comparação com três meses ${ }^{(3)}$. Incremento da satisfação com o tratamento decorre de reduções na sobrecarga social e na interferência em relação às atividades de vida diária, e reflete o impacto psicossocial da terapêutica na vida dos pacientes ${ }^{(4)}$.

No contexto brasileiro, com o fortalecimento do controle social no Sistema Único de Saúde (SUS), iniciaram-se na década de 1990 os primeiros estudos sobre satisfação do usuário dos serviços de saúde ${ }^{(5)}$.

A maioria dos estudos sobre satisfação dos usuários dos serviços de saúde focaliza adesão ao tratamento, acessibilidade, infraestrutura, tempo de espera, relação com os profissionais de saúde e resolutividade ${ }^{(1,6-7)}$. Na atualidade, os gestores dos serviços de saúde já incluíram no planejamento dos serviços, de forma permanente, a avaliação dos indicadores de qualidade relacionados à satisfação dos usuários dos serviços de saúde ${ }^{(8-11)}$.

Por outro lado, os estudos relacionados à avaliação da qualidade da assistência prestada aos usuários com diabetes mellitus (DM) em programa de automonitorização da glicemia capilar ainda são escassos $^{(12-13)}$. Tal avaliação é importante no contexto brasileiro porque este programa foi implantado na rede pública de saúde em 2006.

Diante do exposto, este estudo tem como objetivo avaliar a satisfação dos usuários com DM após oferecimento de um programa educativo para automonitorização da glicemia capilar no domicílio. Estudos de satisfação são relevantes porque oferecem um feedback das intervenções realizadas pela equipe de saúde, proporcionando melhoria dos serviços. Espera-se que os resultados possam contribuir com subsídios para o fortalecimento dos grupos de educação nos serviços de saúde.

\section{MÉTODO}

Estudo quantitativo, descritivo, transversal, realizado em duas Unidades Básicas de Saúde (UBS) do Distrito Oeste da Secretaria Municipal de Saúde de um município do interior paulista, em 2012. Essas Unidades de Saúde foram selecionadas por estarem situadas no Distrito Oeste e serem vinculadas à universidade. A população do estudo foi constituída por 35 pacientes com DM cadastrados no Programa de Automonitorização da Glicemia Capilar (AMGC) no domicílio, que participaram do programa educativo aplicado em um estudo clínico randomizado, realizado em 2011. O Programa de AMGC foi implantado em novembro de 2005, atendendo à Lei Municipal no $10.299 / 04$, que dispõe sobre as normas de proteção à pessoa com DM para garantia de insumos para AMGC no domicílio. O critério de inclusão era ter participado do programa educativo implementado em 2011.

Dos 35 sujeitos que participaram do programa educativo, um foi excluído devido a óbito, dois por não terem registro de telefone no domicilio, dois por mudança de endereço e cidade, e dois que se recusaram a participar do estudo. Assim, a amostra de conveniência foi constituída por 29 indivíduos com DM.

Para obtenção dos dados foram utilizados dois instrumentos: um formulário contendo as variáveis sociodemográficas (sexo, idade, escolaridade, renda familiar, ocupação) e clínicas (tempo de diagnóstico, tratamento para o controle do DM e presença de comorbidades), e um questionário com variáveis qualitativas para avaliar a satisfação dos pacientes em relação ao grupo de educação em diabetes. Entre os instrumentos de avaliação da satisfação em relação aos programas de educação em diabetes disponíveis, destacam-se os questionários. Estes instrumentos possibilitam a abordagem dos aspectos relacionados ao tratamento e à compreensão dos fatores emocionais e sociais relacionados ao atendimento de pessoas com condições crônicas de saúde.

Foi utilizado um questionário elaborado pelos especialistas do International Diabetes Center (IDC), de Minneapolis, Minnesota-EUA, traduzido e adaptado para a língua portuguesa em estudo anterior sobre satisfação dos usuários com $\mathrm{DM}^{(14)}$. O questionário contém oito questões, sendo cinco fechadas e três abertas. Nas questões 
fechadas havia um espaço para justificar a opção sim ou não. As questões abertas abordavam: informações sobre o diabetes que o usuário aprendeu no grupo, quais os sentimentos em relação ao controle do DM, antes e após participar do grupo, e recomendações do grupo de AMGC a outras pessoas com diabetes. Os relatos referentes às respostas abertas, bem como às justificativas das respostas às questões fechadas, foram agrupados por similaridade. Para análise das respostas relacionadas à satisfação do usuário, utilizou-se análise descritiva, com resultados expressos em números absolutos e percentagem.

O recrutamento dos pacientes foi realizado por telefone. Um mês após a primeira aproximação, foi realizada outra ligação telefônica para cada um dos 29 sujeitos, confirmando o dia e horário de sua preferência para a realização da entrevista e assinatura do Termo de Consentimento Livre e Esclarecido. A coleta de dados foi realizada por meio de entrevista dirigida, no domicílio, garantindo-se a privacidade dos sujeitos. As entrevistas foram realizadas no segundo semestre de 2012, com duração média de 30 minutos. Para análise dos dados foi utilizada estatística descritiva. O projeto foi aprovado pelo Comitê de Ética em Pesquisa, Protocolo no 418.

\section{RESULTADOS}

Dos 29 (100\%) pacientes investigados, a maioria era do sexo feminino, na faixa etária predominante de 50 a 69 anos. Quanto à escolaridade a maioria cursou até o ensino fundamental incompleto, apresentava mais de uma comorbidade, com destaque para hipertensão arterial e dislipidemia. No que se refere ao uso de medicamentos, todos faziam uso de insulina no tratamento. Quanto ao número de consultas/ano obteve-se que metade dos pacientes realizou de uma a quatro consultas ao ano.

Quanto à satisfação dos pacientes com DM após a implementação do programa educativo, as respostas obtidas para cada questão aberta investigada foram agrupadas e descritas. O Quadro 1 mostra a distribuição numérica e percentual das respostas obtidas, segundo a frequência expressa em números absolutos e percentagem.

Quadro 1: Distribuição numérica e percentual das respostas obtidas para as questões investigadas. Ribeirão Preto, SP, 2012.

\begin{tabular}{|c|c|}
\hline Respostas & n (\%) \\
\hline \multicolumn{2}{|l|}{ Aprendizado sobre o diabetes no Programa de AMGC no domicilio } \\
\hline Melhor controle da alimentação & $19(65,51)$ \\
\hline Como a doença modifica o relacionamento interpessoal dos pacientes & $9(31 \%)$ \\
\hline Conhecimento dos insumos & $7(24,1)$ \\
\hline Limitações da doença & $4(13,7)$ \\
\hline Exercício físico para o controle da doença & $3(10,3)$ \\
\hline Como lidar com a doença & $3(10,3)$ \\
\hline \multicolumn{2}{|l|}{ Aprendizado sobre o controle do diabetes e o nível de açúcar no sangue } \\
\hline Aumento do conhecimento já existente & $7(24,1)$ \\
\hline Incentivo/Motivação & $7(24,1)$ \\
\hline Controle correto da doença & $4(13,7)$ \\
\hline Controle da alimentação & $4(13,7)$ \\
\hline Conhecimento da doença & $3(10,3)$ \\
\hline Conviver com o diabetes & $3(10,3)$ \\
\hline O grupo não supriu as necessidades & $2(6,8)$ \\
\hline \multicolumn{2}{|l|}{ Aprendizado sobre alimentação e atividade física } \\
\hline Informações suficientes & $8(27,5)$ \\
\hline Incentivo ao exercício físico & $8(27,5)$ \\
\hline Melhora do conhecimento sobre alimentação e atividade física & $5(17,2)$ \\
\hline Incentivo ao plano alimentar & $3(10,3)$ \\
\hline \multicolumn{2}{|l|}{ Motivo da Recomendação do Grupo de AMGC a outras pessoas } \\
\hline Aprendizado & $13(44,8)$ \\
\hline Convívio com outras pessoas que possuem a doença & $9(31)$ \\
\hline Aumento do conhecimento já existente & $6(20,6)$ \\
\hline \multicolumn{2}{|c|}{ Sentimentos em relação ao controle do diabetes antes e após o programa de AMGC } \\
\hline Aumento do controle sobre o diabetes & $9(31)$ \\
\hline Tratamento e aceitação da doença progrediram após o grupo & $7(24,1)$ \\
\hline O grupo proporcionou conhecimento a respeito da doença & $7(24,1)$ \\
\hline Houve o cuidado em relação à doença apenas durante o grupo & $3(10,3)$ \\
\hline Ausência de melhoras em relação ao tratamento & $1(3,4)$ \\
\hline
\end{tabular}

Os resultados obtidos em relação à satisfação dos pacientes com o aprendizado sobre o diabetes no Programa de AMGC no domicilio mostraram que 19
$(65,5 \%)$ referiram informações relacionadas ao melhor controle da alimentação, nove (31\%) à melhora do relacionamento interpessoal e sete $(24,1 \%)$ à melhora do 
conhecimento sobre os insumos disponibilizados, conforme exemplificado nos relatos a seguir:

[...] aprendi como controlar a comida, não comer exagerado e conhecer os grupos alimentares [...] aprendi andar com balinha na bolsa [...] aprendi que não é só controlar o açúcar [...] melhorei minha autoestima [...] aprendi com o exemplo das outras pessoas [...] aprendi sobre o armazenamento da insulina [...] aprendi sobre a técnica e horário de aplicação da insulina [...].

No que se refere ao controle do diabetes e o nível de açúcar no sangue, evidenciou-se que sete $(24,1 \%)$ pacientes referiram aumento do conhecimento já existente, sete $(24,1 \%)$ incentivo e motivação, e quatro $(13,7 \%)$ controle correto da doença. Os relatos a seguir ilustram os benefícios percebidos pelos pacientes.

[...] atualizei meus conhecimentos, fiquei por dentro do assunto [...] me reeduquei $[\ldots]$ eu não tinha conhecimento de muitas coisas lá ditas [...] recebi incentivo para que pudesse caminhar bem o tratamento [...] obtive ânimo para fazer o tratamento corretamente [...] aprendi muito; antes a gente sabia que fazia de forma incorreta, mas mesmo assim continuava fazendo [...].

Em relação aos motivos para recomendação do programa educativo a outras pessoas, obteve-se que 13 $(44,8 \%)$ dos pacientes referiram obtenção de aprendizado e nove $(31 \%)$ convívio com outras pessoas que têm a doença, conforme exemplificado nos relatos a seguir:

[...] aprendi muito e eles também aprenderam [...] aprendizado do que o médico não ensina [...] convivemos e compartilhamos com outras pessoas experiências sobre a doença [...] troca de experiências, educação e paciência [...] maior conhecimento a respeito da doença, além das amizades $[\ldots]$.

Quanto aos sentimentos em relação ao controle do diabetes antes e após a participação no programa educativo, obteve-se que nove $(31 \%)$ referiram aumento do controle sobre o diabetes e sete $(24,1 \%)$ progressão do tratamento e aceitação da doença após o grupo, conforme relatos a seguir.

[...] eu já possuía controle, depois do grupo esse controle aumentou [...] meu controle melhorou, pois antes minha glicemia era um nível elevado, e agora está controlada [...] sentia que se não realizasse o tratamento, iria morrer, então, com o grupo esse sentimento melhorou [...] antes do grupo eu não aceitava o tratamento, agora já aceito melhor [...] depois que participei do grupo fiquei mais animada com o tratamento, pois antes não me controlava $[\ldots]$.

No que se refere à avaliação do programa educativo obteve-se que a maioria $(62,06 \%)$ dos pacientes considerou-o como excelente/satisfatório. Quanto à avaliação da qualidade das informações recebidas, 29 $(100 \%)$ referiram que, além de atender suas necessidades, também o recomendariam a outras pessoas que convivem com a doença.

\section{DISCUSSÃO}

Os dados obtidos acerca da caracterização sociodemográficas dos sujeitos corroboram outro estudo de avaliação de satisfação de pacientes com condições crônicas ${ }^{(14-15)}$.

A análise da satisfação dos pacientes quanto ao aprendizado sobre o diabetes no Programa de AMGC mostra que as necessidades dos pacientes estão relacionadas aos aspectos do tratamento que eles valorizam, sugerindo que o aprendizado se constrói na relação profissional-paciente e no encontro entre necessidades e a oferta de informação. Participar de um programa educativo para automonitorização da glicemia capilar no domicílio possibilitou, segundo os relatos obtidos, que os pacientes se apropriassem do conhecimento oferecido em relação aos insumos, ao autoconhecimento e aos aspectos relacionados à manutenção dos valores glicêmicos e à prevenção de complicações agudas, como, por exemplo, selecionar melhor a alimentação e ter sempre ao alcance da mão um aporte de glicose para situações emergenciais de hipoglicemia ${ }^{(12-13)}$.

Frente a isso, os programas educativos para a automonitorização da glicemia capilar no domicílio devem reforçar a manutenção de registro diário dos hábitos alimentares e do perfil glicêmico. Esse comportamento pode levar os pacientes com DM a refletirem sobre a doença, permitindo uma estratégia de tomada de decisão mais autônoma frente à doença. Estudo controlado randomizado também mostrou que um programa de AMGC é eficaz quando acompanhado de orientações sobre o plano alimentar e de registros da glicemia obtida no domicílio, que permita a sua interpretação(16).

Desse modo, os relatos obtidos em relação à terapia nutricional, componente essencial para o sucesso de um bom controle metabólico, podem constituir um elemento 
importante em programas educativos para avaliar a satisfação dos seus participantes. A obtenção de conhecimento que permite a utilização dos insumos de forma racional, isto é, otimizar o uso de tiras reagentes e adequado manuseio do glicosímetro, também apareceram nos relatos como indicador de avaliação de satisfação percebida pelas pessoas com $\mathrm{DM}^{(16)}$.

Os relatos em relação ao aprendizado sobre o controle e o nível de açúcar no sangue sugerem que negociar alterações das regras do controle do diabetes, propostas pelos profissionais de saúde, possibilita que o paciente se mantenha motivado para adotar as mudanças comportamentais no estilo de vida, na medida em que se percebe no comando de sua vida, o que o torna mais responsável e consciente de suas decisões. Desse modo, a satisfação com o atendimento recebido pode incentivar a prontidão para o autocuidado em diabetes.

Em relação ao controle do DM, 19 (65,5\%) dos pacientes referiram ter controle da doença, em concordância com estudos que evidenciam que a satisfação do paciente é proporcional à adesão ao tratamento e, portanto, à percepção de controle da doença ${ }^{(8)}$.

As práticas de autocuidado constituem um aspecto fundamental dos programas educativos. Nessa direção, o empoderamento do paciente como coparticipante no processo de tomada de decisão em saúde tem sido enfatizado como estratégia para favorecer o controle metabólico. Quando o paciente está bem informado e participa das decisões acerca de seu tratamento por meio do autocuidado apoiado, os sintomas de ansiedade diminuem e a adesão ao tratamento tende a melhorar ${ }^{(17)}$.

A motivação, o incentivo e o ânimo favorecem que os pacientes enfrentem a doença de forma mais independente, além de propiciar a compreensão dos complexos fatores relacionados ao tratamento medicamentoso e não medicamentoso e as complicações agudas e crônicas do $\mathrm{DM}^{(3,14)}$. No entanto, os aspectos mencionados só podem se efetivar quando os pacientes percebem que suas necessidades estão sendo satisfeitas por meio do acolhimento e do fortalecimento do vínculo com o serviço e os profissionais de saúde ${ }^{(14)}$.

Ao analisar os motivos para recomendação do programa educativo a outras pessoas, constatou-se que o programa educativo constituiu um espaço de expressão de necessidades, percepções e sentimentos, permitindo uma aproximação às experiências pessoais com o processo saúde-doença. A satisfação em participar de um grupo educativo levou os pacientes a considerar que essa experiência promove aprendizado, conhecimento e, principalmente, que lidar com a doença crônica, tal como o diabetes, exige paciência, disciplina e espírito de compartilhamento e colaboração mútua.

A satisfação com o programa educativo é um processo reflexivo em torno das práticas de autocuidado, necessárias para o controle da doença. A reflexão se dá dentro do grupo educativo, no qual o indivíduo, por meio de suas características pessoais, sua visão de mundo particular, incluindo interesses, habilidades, desejos e frustrações que podem influenciar o grupo como um todo $^{(12-14)}$. O vínculo do paciente com o profissional e com os demais participantes do grupo são elementos fundamentais para a construção de novas alternativas de manejo da doença.

A análise dos sentimentos em relação ao controle do diabetes antes e após a participação no programa educativo indica que a convivência com um grupo que congrega pessoas com problemas e contextos semelhantes proporciona experiências que podem favorecer o desenvolvimento de um clima terapêutico, como a ajuda no processo de aceitação da doença e do tratamento. Essa situação auxilia os pacientes a quebrarem barreiras, especialmente pela possibilidade de receberem feedback corretivo e sugestões construtivas de outras pessoas que vivenciam os mesmos problemas e encontram as mesmas limitações relacionadas ao manejo da doença e as práticas de autocuidado ${ }^{(6)}$.

Os dados referentes à avaliação do programa educativo corroboram resultados obtidos em estudo realizado em oito hospitais de seis cidades do México, envolvendo 1323 pacientes, que avaliou a satisfação dos pacientes com hipertensão e diabetes. Esse estudo evidenciou que mais da metade dos pacientes estavam satisfeitos com os cuidados recebidos ${ }^{(15)}$.

Outro estudo, realizado em Blumenau-SC, com objetivo de avaliar a associação entre satisfação com o serviço e não adesão ao tratamento em 341 pacientes com DM atendidos no Programa Saúde da Família, evidenciou que $81,7 \%$ dos entrevistados estavam satisfeitos com a estrutura do serviço e o acesso às consultas ${ }^{(18)}$.

A satisfação com os cuidados de saúde por parte dos pacientes com diabetes é de particular importância, uma vez que o cuidado se dá a longo prazo e que há constante interação do paciente com os serviços de saúde em todas as fases de sua condição. Desse modo, enquanto as necessidades e expectativas dos pacientes são satisfeitas, a colaboração no cuidado e na gestão da doença será mantida. 


\section{CONCLUSÕES}

Conclui-se que a maioria dos pacientes considerou o Programa Educativo para AMGC como excelente/satisfatório e reconheceu sua importância para o atendimento de suas necessidades, bem como o recomendaram a outra pessoa. Ao considerar que este é o primeiro estudo no contexto brasileiro que avaliou a satisfação dos usuários de um programa de AMGC, os resultados obtidos oferecem subsídios a futuros estudos, para comparações e aprofundamento da temática. Também podem sugerir estratégias valiosas para

\section{REFERÊNCIAS}

1. Silva LOL, Dias CA, Soares MM, Rodrigues SM. Acessibilidade ao serviço de saúde: percepções de usuários e profissionais de saúde. Cogitare enferm [Internet]. 2011 [acesso em: $31 \mathrm{mar}$ 2015];16(4):654-60. Disponível em:

http://dx.doi.org/10.5380/ce.v16i4.25434.

2. Grøndahl VA, Hall-Lord ML, Karlsson I, Appelgren J, Wilde-

Larsson B. Exploring patient satisfaction predictors in relation to a theoretical model. Int J Health Care Qual Assur.

2013;26(1):37-54.

3. Wermeling PR, Janssen J, Gorter KJ, Beulens JW, Rutten GE. Satisfaction of well-controlled type 2 diabetes patients with three-monthly and six-monthly monitoring. BMC Fam Pract [Internet]. 2013 [acesso em: 31 mar 2015];14:107. Disponível em: http://dx.doi.org/10.1186/1471-2296-14-107.

4. Peyrot M, Rubin RR; STAR 3 Study Group. Treatment satisfaction in the sensor-augmented pump therapy for A1C reduction 3 (STAR 3) trial. Diabet Med [Internet]. 2013 [acesso em: 31 mar 2015];30(4):464-7. Disponível em:

http://dx.doi.org/10.1111/dme.12079.

5. Esperidião MA, Trad LAB. Avaliação de satisfação de usuários: considerações teórico-conceituais. Cad Saude Publica [Internet]. 2006 [acesso em: 31 mar 2015];22(6):1267-76. Disponível em: http://dx.doi.org/10.1590/S0102-311X2006000600016.

6. Gouveia GC, Souza WV, Luna CF, Szwarcwald CL, Souza Junior PRB. Satisfação dos usuários com a assistência de saúde no estado de Pernambuco, Brasil, 2005. Cien Saude Colet [Internet]. 2011 [acesso em: 31 mar 2015];16(3):1849-61. Disponível em: http://dx.doi.org/10.1590/S141381232011000300020 .

7. Cunha ABO, Vieira-da-Silva LM. Acessibilidade aos serviços de saúde em um município do Estado da Bahia, Brasil, em gestão plena do sistema. Cad Saude Publica [Internet]. 2010 [acesso em: 31 mar 2015];26(4):725-37. Disponível em:

http://dx.doi.org/10.1590/S0102-311X2010000400015.

8. Naidu A. Factors affecting patient satisfaction and healthcare Quality. Int. J. Health Care Qual. Assur. 2009;22(4):366-81. 9. Moimaz SAS, Marques JAM, Saliba O, Garbin CAS, Zina LG, Saliba NA. Satisfação e percepção do usuário do SUS sobre o serviço público de saúde. Physis [Internet]. 2010 [acesso em: 31 mar 2015];20(4):1419-40. Disponível em: http://dx.doi.org/10.1590/S0103-73312010000400019. 10. Alrubaiee $L$, Alkaa'ida F. The mediating effect of patient satisfaction in the patients' perceptions of healthcare quality patient trust relationship. International Journal of Marketing Studies [Internet]. 2011 [acesso em: 31 mar 2015];3(1):10327. Disponível em: http://dx.doi.org/10.5539/ijms.v3n1p103. 11. Al-Borie HM, Damanhouri AMS. Patients' satisfaction of service quality in Saudi hospitals: a SERVQUAL analysis. Int J Health Care Qual Assur. 2013;26(1):20-30.

12. Veras VS, Araújo MFM, Rodrigues FFL, Santos MA, Damasceno MMC, Zanetti ML. Assessment of metabolic control among patients in a capillary glucose self-monitoring program. Acta paul. enferm. [Internet]. 2012 [acesso em: 31 mar estruturar o programa educativo para automonitorização, ao considerar que os insumos e protocolos já estão estabelecidos.

Por outro lado, para obter uma compreensão mais abrangente do fenômeno há necessidade de incluir os profissionais de saúde na investigação, o que constituiu em uma limitação do presente estudo. Diante do exposto recomendam-se investimentos para que os programas educativos disponibilizados nos serviços de saúde às pessoas com DM sejam permanentemente avaliados e que utilizem a satisfação como um indicador de avaliação.

2015];25(3):453-8. Disponível em:

http://dx.doi.org/10.1590/S0103-21002012000300021. 13. Veras VS, Teixeira CRS, Santos MA, Torquato MTCG, Rodrigues FFL, Zanetti ML. Glycemic profile of persons with Diabetes mellitus in a home blood glucose self-monitoring program. Texto contexto - enferm. [Internet]. 2014 [acesso em: 31 mar 2015];23(3):609-16. Disponível em:

http://dx.doi.org/10.1590/0104-07072014002610012. 14. Zanetti ML, Otero LM, Biaggi MV, Santos MA, Péres DS, Guimarães FPM. Satisfaction of diabetes patients under follow-up in a diabetes education program. Rev. Latino-Am. Enfermagem [Internet]. 2007 [acesso em: 31 mar 2015];15(4):583-9. Disponível em: http://dx.doi.org/10.1590/S0104-

11692007000400010.

15. Doubova SV, Pérez-Cuevas R, Zepeda-Arias M, FloresHernández $S$. Satisfaction of patients suffering from type 2 diabetes and/or hypertension with care offered in family medicine clinics in Mexico. Salud Publica Mex [Internet]. 2009 [acesso em: 31 mar 2015];51(3):231-9. Disponível em:

http://dx.doi.org/10.1590/S0036-36342009000300014. 16. Schwedes U, Siebolds M, Mertes G. Meal-related structured self-monitoring of blood glucose: effect on diabetes control in non-insulin-treated type 2 diabetic patients. Diabetes Care [Internet]. 2002 [acesso em: 31 mar 2015];25(11):1928-32. Disponível em: http://dx.doi.org/10.2337/diacare.25.11.1928. 17. Mendes EV. As redes de atenção à saúde. Cien Saude Colet [Internet]. 2010 [acesso em: 31 mar 2015];15(5):2297-305. Disponível em: http://dx.doi.org/10.1590/S141381232010000500005.

18. Barros ACM, Rocha MB, Helena ETS. Adesão ao tratamento e satisfação com o serviço entre pessoas com diabetes mellitus atendidas no PSF em Blumenau, Santa Catarina. ACM arq. catarin. med [Internet]. 2008 [acesso em: 31 mar 2015];37(1):54-62. Disponível em:

http://www.acm.org.br/revista/pdf/artigos/536.pdf.

Artigo recebido em 05/08/2013. Aprovado para publicação em 05/11/2014. Artigo publicado em 31/03/2015. 